\title{
MONITORAMENTO DE RESÍDUOS DE MEDICAMENTOS VETERINÁRIOS EM ALIMENTOS E NO AMBIENTE: MODELO PARA AVALIAÇÃO
}

HOFF, Rodrigo Barcellos ${ }^{1}$.

${ }^{1}$ Ministério da Agricultura, Pecuária e Abastecimento.

\section{RESUMO}

trabalho apresenta uma proposta para elencar prioridades na pesquisa de resíduos de fármacos de uso veterinário utilizados em larga escala na produção animal, tanto em alimentos destinados ao consumo humano como no ambiente. Para exemplificar - modelo proposto, adotou-se neste trabalho o caso específico das sulfonamidas, analisando-se as apresentações farmacêuticas disponíveis no mercado veterinário nacional em dois momentos distintos (2007 e 2017). Segundo o modelo proposto, pode-se dizer que o mesmo é adequado para subsidiar políticas regulatórias e delineamentos de experimentos de métodos de monitoramento de resíduos de medicamentos.

Palavras-chave: Sulfas. Análise de resíduos. Plano Nacional de Controle de Resíduos. Avaliação de risco. 


\section{INTRODUÇÃO}

Medicamentos veterinários são mundialmente utilizados para promover a saúde animal, propiciar ganhos econômicos e aumentar a produtividade da indústria de alimentos de origem animal (ACAR; MOULIN, 2006; MAURICIO et al., 2009). Quando o período de carência não é cumprido, ocorre a permanência de resíduos destes medicamentos nos tecidos destinados à produção de alimentos (AERTS et al., 1995; STRUCIŃSKI et al., 2007). A questão dos resíduos de medicamentos veterinários (RMVs) acarretou o desenvolvimento de legislações e regulamentações próprias tanto no âmbito nacional quanto no internacional, com o propósito de propor e, posteriormente, harmonizar valores de limites máximos de resíduo (LMR) para as diversas combinações fármaco/matriz. O estudo dos potenciais efeitos da ingestão de alimentos contendo quantidades acima do LMR foi e segue sendo uma área de pesquisa de grande relevância, já que diz respeito diretamente à saúde pública, bem como às relações comerciais internacionais. O controle regulatório de contaminantes químicos em alimentos expandiu-se dramaticamente nas últimas décadas, fazendo da área de análise destes resíduos um importante fator a ser considerado no comércio internacional de commodities (QUEIROZ MAURICIO; LINS, 2012).

Mais recentemente, o foco de estudo a respeito dos RMVs tem se voltado para o impacto destes compostos sobre o ambiente (BLACKWELL et al., 2004; BLACKWELL et al., 2007; BOXALL, 2004; BOXALL; LONG, 2005; GARCÍA-GALÁN et al., 2009). Após a administração, estes fármacos são liberados no ambiente através de processos de excreção dos animais, sendo que estas substâncias podem migrar para distintos recursos hídricos. Vários estudos têm citado a ocorrência de RMVs em águas superficiais, lençóis freáticos e efluentes de estações de tratamento (DÍAZ-CRUZ et al., 2008; DONG et al., 2013; FERRER et al., 2010; GIBS et al., 2013). Muitos trabalhos têm relatado ações sobre espécies não-alvo que incluem disrupção endócrina, inibição reprodutiva e até mesmo respostas em nível de ecossistema (BOXALL et al., 2003; BOXALL, 2004; BRASCHI et al., 2013; CAPLETON et al., 2006; SANTOS et al., 2013; WALL; STRONG, 1987). 
Considerando as lacunas existentes no que se refere aos dados de utilização e frequência de utilização de fármacos veterinários, faz-se necessário propor modos de classificação e priorização destes compostos baseados em seu uso, distribuição e perfil toxicológico.

As sulfonamidas constituem a primeira classe de agentes anti-infecciosos descobertos na terapêutica. Seu uso ainda é muito frequente na medicina veterinária, na área de produção animal e principalmente como profilático de infecções na produção em larga escala de aves e suínos (HOFF et al., 2015).

No presente estudo, utilizamos o exemplo das sulfonamidas, uma classe de compostos antibacterianos amplamente utilizada na medicina veterinária, para propor a construção de um modelo preditivo para elencar prioridades na área de RMVs, seja sob o ponto de vista da presença de resíduos em alimentos como também no ambiente. Para tanto, traçamos o perfil das sulfonamidas disponíveis no mercado veterinário brasileiro em dois períodos distintos (2007 e 2017) e aplicou-se o modelo sobre este cenário.

\section{MATERIAL E MÉTODOS}

A pesquisa dos medicamentos de uso veterinário contendo sulfas foi realizada através de revisão bibliográfica, análise de bulas e rótulos, pesquisa através da Internet e em bancos de dados oficiais do MAPA (Ministério da Agricultura, Pecuária e Abastecimento) e pesquisa em casas de comércio agropecuário $(n=27)$. Os dados estatísticos foram obtidos através do uso de software comercial $\left(\right.$ Excel $\left.^{\circledR}\right)$.

\section{RESULTADOS E DISCUSSÃO}

O mercado de medicamentos veterinários no Brasil é um dos maiores do mundo e encontrase em expansão. A regulamentação deste mercado, desde os aspectos de registro e licença para produção até a análise fiscal, é atribuição do MAPA (CARNEIRO; KANEENE, 2017; SINDAN, 2017). A Figura 1 mostra a evolução do faturamento deste segmento no Brasil no período 2004-2012 (medicamentos em geral) e no período 2007-2012 (somente a fração correspondente aos antimicrobianos). O Brasil adota um valor de LMR de $100 \mu \mathrm{g} / \mathrm{kg}$ para resíduos de sulfonamidas em tecidos como músculo, fígado e rim de diversas espécies animais (bovinos, suínos, equinos e aves) (ANVISA, 2012). 


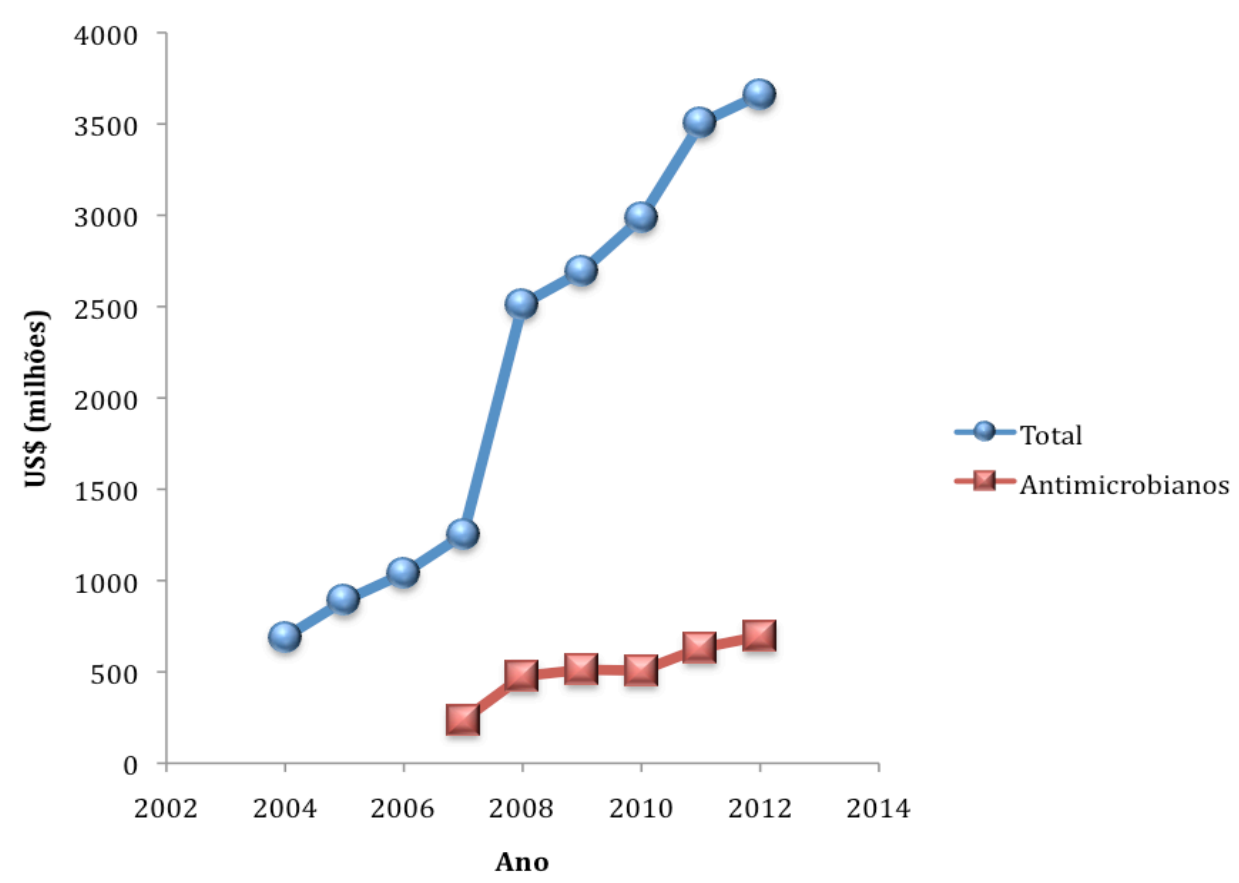

Figura 1 - Faturamento do mercado veterinário brasileiro. Faturamento total (em milhões de doláres) no período 2004-2012: círculos azuis. Faturamento com venda de antimicrobianos (em milhões de dólares) no período 2007-2012: quadrados vermelhos.

O uso de fármacos veterinários no Brasil é de difícil mensuramento, dado que não são encontradas informações sobre quantidades comercializadas. Somente dados de volume de vendas são disponibilizados pelo Sindicato Nacional da Indústria de Produtos Para Saúde Animal (SINDAN, 2016).

No ano de 2007, estavam disponíveis no Brasil 136 medicamentos de uso veterinário contendo sulfonamidas (Tabela 1). Em 2017, foram encontrados 93 produtos disponíveis contendo uma ou mais sulfonamidas como princípios ativos (Tabela 2). Foram analisados os seguintes fatores: sulfonamida(s) presente(s) na formulação; concentração dos princípios ativos; outros fármacos associados; forma farmacêutica; espécies animais com indicação de uso; período de carência para o consumo de produtos de origem animal. 
Tabela 1 - Perfil das apresentações de sulfas no Brasil em 2007.

\begin{tabular}{|c|c|c|c|c|c|c|c|c|c|c|c|c|c|}
\hline \multirow[b]{2}{*}{ SULFONAMIDA } & \multirow{2}{*}{$\begin{array}{l}\text { QUANTIDADE DE } \\
\text { APRESENTAÇÕES }\end{array}$} & \multicolumn{12}{|c|}{ FORMAS FARMACÊUTICAS } \\
\hline & & INJ & SOL & SUS & PÓ & POM & CMP & INF & ERR & PRE & OUT & total & $\%$ \\
\hline Sulfadiazina & 32 & 7 & 2 & 6 & 9 & 1 & 1 & 1 & --- & 3 & 2 & 32 & 23,5 \\
\hline Sulfametazina & 16 & 3 & 3 & 1 & 2 & --- & 1 & --- & 1 & 5 & --- & 16 & 11,7 \\
\hline Sulfametoxazol & 15 & 3 & 4 & 1 & 5 & --- & 2 & --- & --- & --- & --- & 15 & 11,0 \\
\hline Ftalilsulfatiazol & 13 & --- & 2 & 1 & 9 & --- & --- & --- & -- & -- & 1 & 13 & 9,6 \\
\hline Sulfaquinoxalina & 13 & --- & 8 & --- & 4 & --- & --- & --- & -- & 1 & --- & 13 & 9,6 \\
\hline Sulfanilamida & 12 & --- & --- & --- & --- & 9 & --- & 1 & 1 & -- & 1 & 12 & 8,8 \\
\hline Sulfaguanidina & 9 & --- & 1 & 1 & 6 & --- & 1 & --- & -- & -- & --- & 9 & 6,6 \\
\hline Sulfaclorpiridazina & 5 & --- & 2 & --- & 3 & --- & --- & --- & -- & --- & --- & 5 & 3,7 \\
\hline Sulfadimetoxina & 5 & 2 & 1 & -- & 1 & --- & 1 & --- & --- & -- & -- & 5 & 3,7 \\
\hline Sulfacetamida & 4 & 2 & 1 & --- & --- & --- & --- & --- & --- & -- & 1 & 4 & 2,9 \\
\hline Sulfamerazina & 3 & --- & -- & --- & 3 & --- & --- & --- & -- & --- & --- & 3 & 2,2 \\
\hline Sulfatiazol & 3 & 1 & --- & 1 & -- & --- & 1 & --- & -- & -- & -- & 3 & 2,2 \\
\hline Sulfadoxina & 2 & 1 & 1 & --- & --- & --- & --- & --- & -- & -- & --- & 2 & 1,5 \\
\hline Sulfafurazol & 1 & --- & -- & --- & 1 & --- & --- & --- & -- & -- & --- & 1 & 0,7 \\
\hline Sulfaisoxazol & 1 & --- & --- & --- & 1 & --- & --- & --- & --- & --- & --- & 1 & 0,7 \\
\hline Sulfametilpirimidina & 1 & 1 & --- & --- & --- & --- & --- & --- & --- & --- & --- & 1 & 0,7 \\
\hline Sulfametoxipiridazina & 1 & 1 & -- & --- & --- & --- & --- & --- & -- & -- & --- & 1 & 0,7 \\
\hline Total & 136 & 21 & 25 & 11 & 44 & 10 & 7 & 2 & 2 & 9 & 5 & 136 & \\
\hline Porcentagem & 100 & 15 & 18 & 8 & 32 & 7 & 5 & 2 & 2 & 7 & 4 & & \\
\hline
\end{tabular}

INJ (injetável), SOL (solução de uso oral), SUS (suspensão de uso oral), PÓ (pó para uso oral), POM (pomada), CMP (comprimido), INF (infusão intramamária), ERR (errino), PRE (premix), OUT (outros). 
Tabela 2 - Perfil das apresentações de sulfas no Brasil em 2017.

\begin{tabular}{|c|c|c|c|c|c|c|c|c|c|c|c|c|c|}
\hline \multirow[b]{2}{*}{ SULFONAMIDA } & \multirow{2}{*}{$\begin{array}{l}\text { QUANTIDADE DE } \\
\text { APRESENTAÇÕES }\end{array}$} & \multicolumn{5}{|c|}{ FORMAS FARMACÊUTICAS } & \multirow[b]{2}{*}{ CMP } & \multirow[b]{2}{*}{ INF } & \multirow[b]{2}{*}{ ERR } & \multirow[b]{2}{*}{ PRE } & \multirow[b]{2}{*}{ OUT } & \multirow[b]{2}{*}{ total } & \multirow[b]{2}{*}{$\%$} \\
\hline & & INJ & SOL & SUS & PÓ & POM & & & & & & & \\
\hline Sulfadiazina & 24 & 9 & 3 & --- & 4 & --- & --- & 2 & --- & --- & 6 & 24 & 17,6 \\
\hline Sulfametazina & 16 & 1 & 4 & --- & 10 & --- & --- & --- & 1 & --- & --- & 16 & 11,8 \\
\hline Sulfametoxazol & 16 & 3 & 4 & 2 & 5 & --- & 2 & --- & --- & --- & --- & 16 & 11,8 \\
\hline Ftalilsulfatiazol & 5 & --- & --- & --- & 4 & --- & 1 & --- & --- & --- & --- & 5 & 3,7 \\
\hline Sulfaquinoxalina & 14 & --- & 4 & --- & 10 & --- & --- & --- & --- & --- & --- & 14 & 10,3 \\
\hline Sulfanilamida & 4 & --- & --- & --- & --- & 2 & --- & 1 & 1 & --- & --- & 4 & 2,9 \\
\hline Sulfaguanidina & 2 & --- & --- & --- & 1 & --- & 1 & --- & --- & --- & --- & 2 & 1,5 \\
\hline Sulfaclorpiridazina & 3 & --- & --- & --- & 3 & --- & --- & --- & --- & --- & --- & 3 & 2,2 \\
\hline Sulfadimetoxina & 4 & 2 & 1 & --- & 1 & --- & --- & --- & --- & --- & --- & 4 & 2,9 \\
\hline Sulfacetamida & 1 & 1 & --- & --- & --- & --- & --- & --- & --- & --- & --- & 1 & 0,7 \\
\hline Sulfamerazina & 1 & --- & --- & --- & --- & --- & 1 & --- & --- & --- & --- & 1 & 0,7 \\
\hline Sulfatiazol & 0 & --- & --- & --- & --- & --- & --- & --- & --- & --- & --- & 0 & 0 \\
\hline Sulfadoxina & 2 & 2 & --- & --- & --- & --- & --- & --- & --- & --- & --- & 2 & 1,5 \\
\hline Sulfafurazol & 0 & --- & --- & --- & --- & --- & --- & --- & --- & --- & --- & 0 & 0 \\
\hline Sulfaisoxazol & 0 & --- & --- & --- & --- & --- & --- & --- & --- & --- & --- & 0 & 0 \\
\hline Sulfametilpirimidina & 0 & --- & --- & --- & --- & --- & --- & -- & --- & --- & --- & 0 & 0 \\
\hline Sulfametoxipiridazina & 1 & --- & --- & --- & 1 & --- & --- & --- & --- & --- & --- & 1 & 0,7 \\
\hline Total & 93 & 18 & 16 & 2 & 39 & 2 & 5 & 3 & 2 & 0 & 6 & 93 & \\
\hline Porcentagem & 100 & 13 & 12 & 1,5 & 29 & 1,5 & 3,7 & 2,2 & 1,5 & 0 & 4,4 & & \\
\hline
\end{tabular}

INJ (injetável), SOL (solução de uso oral), SUS (suspensão de uso oral), PÓ (pó para uso oral), POM (pomada), CMP (comprimido), INF (infusão intramamária), ERR (errino), PRE (premix), OUT (outros).

Ocorreu uma significativa dimuição no número de produtos, aproximadamente $32 \%$, sendo que algumas sulfas foram retiradas do mercado e hoje já não se encontram disponíveis para comercialização, como é o caso de sulfatiazol, sulfafurazol, sulfaisoxazol e sulfametilpirimidina. A Tabela 3 mostra um quadro comparativo entre os dois cenários avaliados. 
Tabela 3 - Comparação das apresentações contendo sulfonamidas disponíveis nos anos de 2007 e 2017.

\begin{tabular}{lllll}
\hline Sulfa & $\begin{array}{l}2007 \\
\text { (número) }\end{array}$ & $2007(\%)$ & $\begin{array}{l}2017 \\
\text { (número) }\end{array}$ & $2017(\%)$ \\
\hline Sulfadiazina & 32 & 23,5 & 24 & 25,8 \\
Sulfametazina & 16 & 11,8 & 16 & 17,2 \\
Sulfametoxazol & 15 & 11,0 & 16 & 17,2 \\
Ftalilsulfatiazol & 13 & 9,6 & 5 & 5,4 \\
Sulfaquinoxalina & 13 & 9,6 & 14 & 15,1 \\
Sulfanilamida & 12 & 8,8 & 4 & 4,3 \\
Sulfaguanidina & 9 & 6,6 & 2 & 2,2 \\
Sulfaclorpiridazina & 5 & 3,7 & 3 & 3,2 \\
Sulfadimetoxina & 5 & 3,7 & 4 & 4,3 \\
Sulfacetamida & 4 & 2,9 & 1 & 1,1 \\
Sulfamerazina & 3 & 2,2 & 1 & 1,1 \\
Sulfatiazol & 3 & 2,2 & 0 & 0,0 \\
Sulfadoxina & 2 & 1,5 & 2 & 2,2 \\
Sulfafurazol & 1 & 0,7 & 0 & 0,0 \\
Sulfaisoxazol & 1 & 0,7 & 0 & 0,0 \\
Sulfametilpirimidina & 1 & 0,7 & 0 & 0,0 \\
Sulfametoxipiridazina & 1 & 0,7 & 1 & 1,1 \\
\hline Total & 136 & & 93 & \\
\hline
\end{tabular}

No caso do sulfatiazol, cabe esclarecer que este fármaco segue sendo disponiblizado na forma de ftalilsulfatiazol, com cinco apresentações disponíveis em 2017. Em 2007, havia 16 produtos contendo sulfatiazol ou ftalilsulfatiazol. O ftalilsulfatiazol é um pró-fármaco, elaborado para que não sofra absorção em nível estomacal ou nas primeiras porções intestinais. O radical ftalil é liberado por clivagem mediada pela microbiota intestinal, liberando o sulfatiazol.

A Figura 2 mostra um quadro comparativo do número de formulações comerciais disponíveis nos dois cenários temporais. 


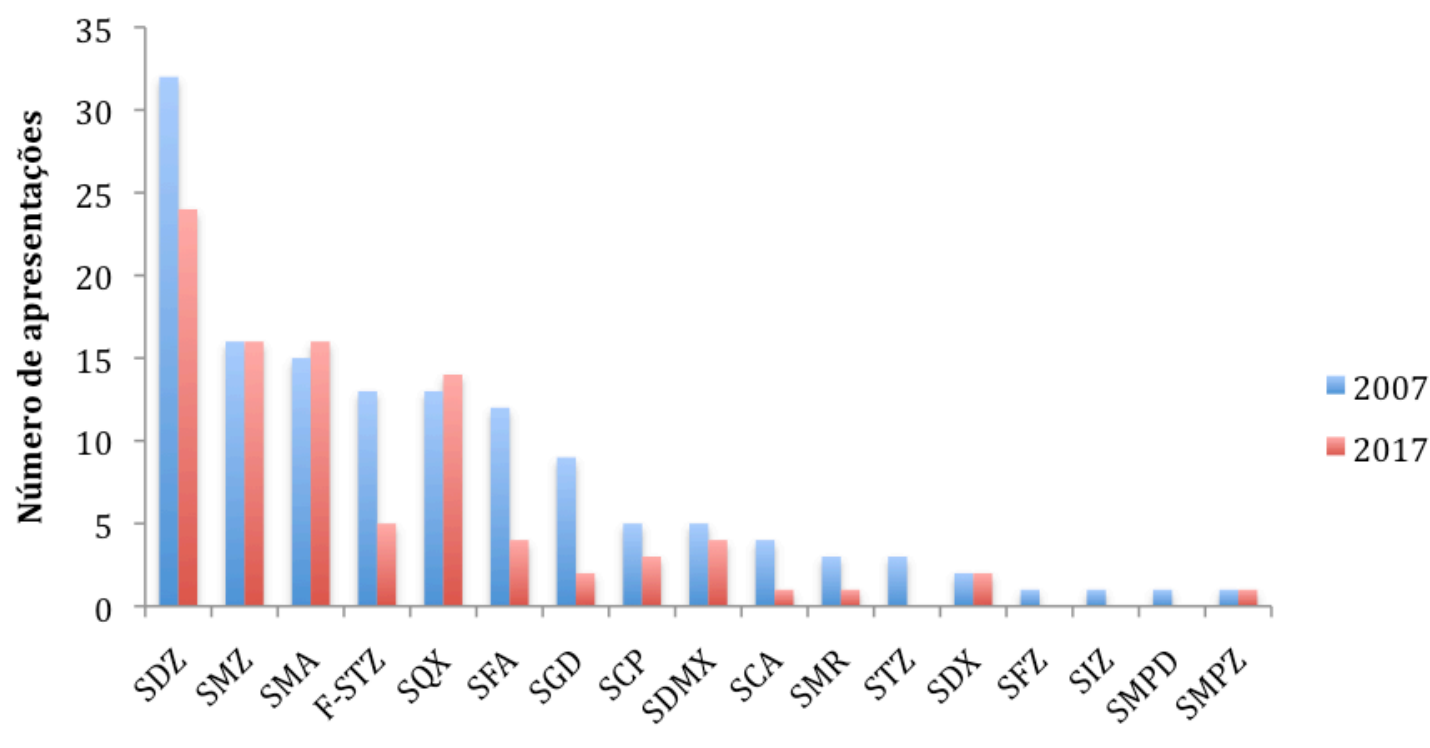

Sulfonamida

Figura 2 - Número de apresentações por sulfonamida disponíveis no mercado veterinário brasileiro em 2007 e em 2017. SDZ (sulfadiazina), SMZ (sulfametazina), SMA (sulfametoxazol), F-STZ (ftalilsulfatiazol), SQX (sulfaquinoxalina), SFA (sulfanilamida), SGD (sulfaguanidina), SCP (sulfaclorpiridazina), SDMX (sulfadimetoxina), SCA (sulfacetamida), SMR (sulfamerazina), STZ (sulfatiazol), SDX (sulfadoxina), SFZ (sulfafurazol), SIZ (sulfaisoxazol), SMPD (sulfametilpirimidina) e SMPZ (sulfametoxipiridazina).

As concentrações do princípio ativo na forma farmacêutica variaram desde $0.1 \%$ (sulfadiazina de prata em spray) até $80 \%$ (sulfadiazina em pó para uso oral). As formas farmacêuticas são variadas, com predominância de pós para uso oral, soluções de uso oral e injetáveis.

As associações medicamentosas representaram 89\% do total em 2007 e 82\% em 2017. Dentre os fármacos de outras classes, a associação mais utilizada é com trimetoprima. Mais da metade (54\% em 2007 e 63\% em 2013) das associações contém trimetoprima. Há ocorrência de associações de duas, três e quatro sulfas em uma mesma forma farmacêutica. A Tabela 4 mostra o perfil das associações de sulfas nos dois períodos do estudo. 
Tabela 4 - Características das apresentações contendo sulfas em associação com outros fármacos: comparação entre os anos de 2007 e 2017.

\begin{tabular}{lllll}
\hline Associações & $\begin{array}{l}2007 \\
\text { (número) }\end{array}$ & \% total & $\begin{array}{l}2017 \\
\text { (número) }\end{array}$ & \% total \\
\hline Apresentações & 105 & 89 & 76 & 82 \\
\hline Duas sulfas & 11 & 11 & 8 & 10 \\
Três sulfas & 1 & 1 & 5 & 7 \\
Quatro sulfas & 2 & 2 & 0 & 0 \\
Trimetoprima & 57 & 54 & 48 & 63 \\
Outras & 34 & 32 & 15 & 20 \\
\hline
\end{tabular}

As indicações de uso das apresentações abrangem praticamente todas as espécies animais produtoras de alimento (com exceção de pescado) bem como de animais de estimação (pets). O período de carência para retirada da medicação em animais produtores de alimentos foi analisado com base em estudo de bulas. Os períodos variam enormemente, mesmo para formas farmacêuticas semelhantes. Muitas bulas não apresentam nenhuma informação referente ao período de carência, mesmo em casos nos quais o medicamento é indicado para espécies produtoras de alimentos.

Para estabelecer um modelo para priorização de sulfas a serem monitoradas em alimentos e no meio-ambiente, se propõe à adoção do modelo exposto na Tabela 5 . Critérios de pontuação numérica simples foram utilizados, atribuindo valores a determinados parâmetros que devem ser observados para elencar, em escala de prioridades, os fármacos que devem ser monitorados dentro do Plano Nacional de Controle de Resíduos (QUEIROZ MAURICIO; LINS, 2012), e avaliados quanto ao seu impacto na microbiota de solo e mananciais de água.

Para as sulfas, o potencial de permanência de resíduos em alimentos é proporcional ao período de permanência do fármaco no organismo (1/2 vida) e inversamente proporcional à extensão do processo de metabolização do fármaco. Deste modo, para o sulfatiazol, que geralmente é utilizado na forma de pró-fármaco (ftalilsulfatiazol) e que é excretado de forma inalterada e com absorção desprezível, o potencial de permanência de resíduos em alimentos é bastante baixo. Não obstante, para avaliações do impacto ambiental de resíduos de sulfas, estas relações devem ser invertidas. Assim, o sulfatiazol, sendo amplamente 
excretado em curto intervalo de tempo, possui preocupante papel na migração de fármacos ao ambiente.

Tabela 5 - Proposta de critérios de pontuação para priorização de sulfonamidas a serem monitoradas em alimentos e no meio-ambiente.

\begin{tabular}{|c|c|c|c|c|}
\hline Critério & Pontuações & Alimentos & Ambiente & Peso \\
\hline Número de apresentações disponíveis: um grande & $>30$ & 5 & 5 & \multirow{5}{*}{1} \\
\hline número de apresentações pode ser levado em conta & $20-30$ & 4 & 4 & \\
\hline como um indicador subjetivo de "popularidade" do & $10-20$ & 3 & 3 & \\
\hline fármaco, embora sejam necessários dados de uso e & $05-10$ & 2 & 2 & \\
\hline $\begin{array}{l}\text { volume de vendas por substância para racionalizar } \\
\text { devidamente o processo }\end{array}$ & $<05$ & 1 & 1 & \\
\hline \multirow{4}{*}{$\begin{array}{l}\text { Espécies indicadas: de acordo com a(s) espécie(s) em } \\
\text { que o fármaco é utilizado, tem-se um perfil de manejo } \\
\text { e administração que é de extrema relevância para o } \\
\text { potencial de resíduos ou impacto ambiental }\end{array}$} & Aquacultura & 5 & 5 & \multirow{4}{*}{3} \\
\hline & $\begin{array}{l}\text { Tratamento } \\
\text { coletivo }\end{array}$ & 4 & 4 & \\
\hline & & 3 & 3 & \\
\hline & Animais & 3 & 3 & \\
\hline \multirow{7}{*}{$\begin{array}{l}\text { Dados farmacológicos: substâncias de baixa absorção, } \\
\text { destinadas à efeito na luz intestinal, como o sulfatiazol, } \\
\text { por exemplo, tem baixo potencial de resíduos em } \\
\text { alimentos, mas alto índice para avaliação de impacto } \\
\text { ambiental }\end{array}$} & $\begin{array}{l}\text { Baixa } \\
\text { metabolização }\end{array}$ & 3 & 3 & \multirow{3}{*}{2} \\
\hline & $\begin{array}{l}\text { Média } \\
\text { metabolização }\end{array}$ & 2 & 2 & \\
\hline & $\begin{array}{l}\text { Alta } \\
\text { metabolização }\end{array}$ & 1 & 1 & \\
\hline & $\begin{array}{l}\text { Ação curta } \\
\text { Ação }\end{array}$ & 1 & 4 & \\
\hline & intermediária & 2 & 3 & 2 \\
\hline & Ação longa & 3 & 2 & \\
\hline & Ação ultra-longa & 4 & 1 & \\
\hline \multirow{4}{*}{$\begin{array}{l}\text { Forma farmacêutica: dependendo da forma } \\
\text { farmacêutica, há maior ou menor probabilidade de } \\
\text { permanência do fármaco em tecidos }\end{array}$} & Injetáveis & 5 & 2 & \multirow{4}{*}{1} \\
\hline & Rações & 4 & 3 & \\
\hline & Solução oral & 4 & 3 & \\
\hline & Errinos, pomadas & 1 & 2 & \\
\hline \multirow[t]{4}{*}{ Genotoxicidade e teratogenicidade } & $\begin{array}{ll}\text { Alto } & \text { ou } \\
\text { desconhecido }\end{array}$ & 5 & 5 & \multirow{4}{*}{3} \\
\hline & Médio & 4 & 4 & \\
\hline & Baixo & 3 & 3 & \\
\hline & Insignificante & 1 & 1 & \\
\hline \multirow[t]{4}{*}{ Potencial alergênico } & $\begin{array}{l}\text { Alto ou } \\
\text { desconhecido }\end{array}$ & 5 & 5 & \multirow{4}{*}{2} \\
\hline & Médio & 4 & 4 & \\
\hline & Baixo & 3 & 3 & \\
\hline & Insignificante & 1 & 1 & \\
\hline
\end{tabular}

Baseado na proposta de pontuação que consta na Tabela 5, as sulfas foram elencadas de acordo com sua pontuação para estudos de permanência de resíduos em alimentos. Estes resultados estão computados na Tabela 6. 
Tabela 6 - Pontuação das sulfonamidas de acordo com os critérios propostos.

\begin{tabular}{|c|c|c|c|c|c|c|c|c|c|c|c|c|c|}
\hline Critério & SDZ & STZ & SMZ & SMA & SQX & SFA & SGD & SCP & SDMX & SCA & SMR & SDX & PESO \\
\hline Apresentações & 5 & 3 & 3 & 3 & 3 & 3 & 2 & 2 & 2 & 1 & 1 & 1 & 1 \\
\hline Indicações & 4 & 4 & 4 & 4 & 4 & 4 & 4 & 4 & 4 & 4 & 4 & 4 & 3 \\
\hline $\begin{array}{l}\text { Farmacologia } \\
\text { (metabolizaçao) }\end{array}$ & 3 & 3 & 3 & 3 & 3 & 3 & 3 & 3 & 1 & 3 & 2 & 3 & 2 \\
\hline $\begin{array}{l}\text { Farmacologia } \\
\text { (1/2 vida) }\end{array}$ & 2 & 1 & 3 & 1 & 2 & 1 & 1 & 2 & 3 & 2 & 2 & 4 & 2 \\
\hline $\begin{array}{l}\text { Forma } \\
\text { farmacêutica }\end{array}$ & 4 & 4 & 4 & 4 & 4 & 1 & 4 & 4 & 4 & 4 & 4 & 5 & 1 \\
\hline $\begin{array}{l}\text { Genotoxicidade } \\
\text { Teratogenicidade }\end{array}$ & 1 & 5 & 5 & 1 & 3 & 1 & 1 & 1 & 1 & 1 & 3 & 1 & 3 \\
\hline Alergenicidade & 3 & 3 & 3 & 3 & 3 & 3 & 3 & 3 & 3 & 3 & 3 & 3 & 2 \\
\hline Total & 40 & 48 & 52 & 36 & 44 & 33 & 35 & 37 & 35 & 36 & 40 & 41 & \\
\hline
\end{tabular}

SDZ (sulfadiazina), STZ (sulfatiazol), SMZ (sulfametazina), SMA (sulfametoxazol), SQX (sulfaquinoxalina), SFA (sulfanilamida), SGD (sulfaguanidina), SCP (sulfaclorpiridazina), SDMX (sulfadimetoxina), SCA (sulfacetamida), SMR (sulfamerazina), SDX (sulfadoxina).

Para o critério de número de apresentações, adotamos faixas de valores aos quais são atribuidos valores de 1 a 5 . Este critério se baseia na inferência de que o número de apresentações disponíveis é diretamente proporcional ao volume utilizado. Esta correlação nem sempre é verdadeira, como por exemplo, no caso de medicamentos inovadores detentores de patente. No entanto, face à ausência de dados de comercialização, pode ser considerada como uma aproximação. Nos demais critérios, foram utilizados dados obtidos pelas próprias bulas dos medicamentos, bem como dados farmacológicos e toxicológicos (BRACKETT et al., 2004; CRIBB et al., 1996; DREWS, 2000; GHIMIRE et al., 2013; NEU, 1992; PARK; KWAK, 2012; VAN DER VEN et al., 1997; VREE et al., 1985; VREE; VAN DER VEN, 1997).

De acordo com os critérios propostos e o peso de cada um na pontuaçao geral, elencou-se a sulfametazina como a sulfa de maior impacto. Este modelo apresenta concordância com os resultados de monitoramento de resíduos de sulfas em alimentos, no qual a sulfametazina apresenta o maior número de resultados não-conformes (LEITE; JOKL, 1996). Em segundo lugar, está o sulfatiazol. Conforme comentado anteriormente, este fármaco tem grande potencial de contaminação ambiental e aspectos de sua toxicidade e do risco ambiental associado estão bem documentados (Jl et al., 2012; LIN et al., 2013). A sulfaquinoxalina aparece na terceira pontuação mais alta. Este fármaco, ao lado da sulfametazina, apresenta uma das maiores incidências em amostras não-conformes analisadas pelo MAPA (LEITE; JOKL, 1996; QUEIROZ MAURICIO; LINS, 2012). Entretanto, em geral são amostras de fígado 
de aves. As aves não apresentam um importante mecanismo de metabolização para SQX, que, no entanto, é muito signifcativo para outras espécies (HOFF et al., 2012). Deste modo, não somente se deve levar em conta a molécula original, bem como seus metabólitos e produtos de degradação.

Embora se disponha de dados farmacológicos consistentes para muitas sulfas, como sulfametoxazol e sulfadiazina, ainda restam muitas lacunas de informações. Dados de comercialização, por exemplo, não puderam ser utilizados por serem virtualmente inexistentes. Informações toxicológicas também existem somente para alguns compostos. A ferramenta proposta necessita de diversos dados para que possa fornecer predições mais robustas e confiáveis. Conclui-se que a comunidade científica brasileira e o público em geral necessitam com urgência de acesso transparente a informações sobre o real cenário da utilização de fármacos de uso veterinário no Brasil, de modo a poder avaliar corretamente os impactos ambientais e alimentares de RMVs.

\section{CONCLUSÃO}

A adoção de modelos racionais para a elaboração de políticas regulatórias e também para o desenvolvimento de ferramentas analíticas de monitoramento é crucial para a otimização do uso de recursos, tanto em termos de custos laboratoriais como em recursos humanos. $\mathrm{O}$ modelo apresentado neste trabalho poderá se tornar uma alternativa interessante neste sentido, permitindo elencar as substâncias com base em um sistema de pontuação simples e de fácil aplicação. Cabe ressaltar, entretanto, que o Brasil precisa com urgência produzir os dados que irão alimentar modelos como este, que são de extrema importância, não somente do ponto de vista estratégico como são fundamentais do ponto de vista da saúde pública e da farmacovigilância. Atualmente, não estão publicamente disponíveis dados sobre o consumo de fármacos de uso veterinário no Brasil que permitam estabelecer critérios técnicos objetivos para subsidiar a regulamentação e a pesquisa científica nessa área.

Em relação ao exemplo utilizado para avaliar o modelo, podemos concluir que as sulfonamidas disponíveis no mercado veterinário brasileiro têm apresentado perfil mais homogêneo nos últimos anos, focando-se em produtos destinados para suínos e aves. 
Em síntese, o modelo proposto para priorização será adequado uma vez que os dados necessários sejam disponibilizados: evidenciamos claramente a necessidade urgente de maiores pesquisas e criação de bases de dados sobre o uso de medicamentos veterinários no Brasil.

\title{
VETERINARY DRUGS MONITORING IN FOOD AND ENVIRONMENT: AN EVALUATION MODEL
}

\section{ABSTRACT}

T he purpose of this work is to present a proposal for electing priorities in the search of residual drugs of veterinary use in large-scale animal production, which can produce residue levels in both food and the environment. To illustrate the proposed model, the specific case of sulfonamides was used, analyzing all pharmaceutical presentations available in the Brazilian veterinarian market in two distinct moments (2007 and 2017). The proposed model has showed to be an adequate tool to support regulatory decisions and to be applied in the development of methods for veterinary drug residue monitoring.

Keywords: Sulfonamides. Residue analysis. Veterinary drugs. National Plan of Residue Control. Risk evaluation.

\section{MONITOREO DE RESIDUOS DE DROGAS VETERINARIAS EN ALIMENTOS Y EN EL AMBIENTE: MODELO DE EVALUACIÓN}

\section{RESUMEN}

\begin{abstract}
- I propósito de este trabajo es presentar una propuesta para priorización de sustancias - en la investigación de drogas veterinarias utilizadas en la producción animal en gran - escala, que puede producir presencia de residuos en los alimentos y el ambiente. Para ejemplificar el modelo, se utilizó el caso específico de las sulfonamidas, mediante el análisis de todas las preparaciones disponibles en el mercado veterinario brasileño en dos escenarios distintos (2007 y 2017). Ha sido posible concluir que el modelo propuesto es adecuado para la elaboración de las políticas regulatorias y en el desarrollo de métodos de monitoreo de residuos de medicamentos.
\end{abstract}

Palabras clave: Sulfonamidas. Análisis de residuos. Drogas veterinarias. Evaluación de riesgo. 


\section{REFERÊNCIAS}

ACAR, J. F.; MOULIN, G. Antimicrobial resistance at farm level. OIE Revue Scientifique et Technique, v. 25, n. 2, p. 775-792, 2006.

AERTS, M. M. L.; HOGENBOOM, A. C.; BRINKMAN, U. A. T. Analytical strategies for the screening of veterinary drugs and their residues in edible products. Journal of

Chromatography B: Biomedical Applications, v. 667, n. 1, p. 1-40, 1995.

ANVISA - AGÊNCIA NACIONAL DE VIGILÂNCIA SANITÁRIA. Resolução RDC № 53, de 2 de outubro de 2012. Diário Oficial da União, n. 192, Seção 1, p. 47, 03 de out. 2012. Disponível em: <http://portal.anvisa.gov.br/documents/33916/388704/RDCN53.pdf/4f53a37a-03a94b8e-80eb-a31cc01964fa>.

BLACKWELL, P. A.; LÜTZHOFT, H. H.; MA, H.; et al. Ultrasonic extraction of veterinary antibiotics from soils and pig slurry with SPE clean-up and LC-UV and fluorescence detection. Talanta, v. 64, n. 4, p. 1058-1064, 2004.

BLACKWELL, P. A.; KAY, P.; BOXALL, A. B. A. The dissipation and transport of veterinary antibiotics in a sandy loam soil. Chemosphere, v. 67, n. 2, p. 292-299, 2007.

BOXALL, A. B. A.; KOLPIN, D. W.; HALLING-SORENSEN, B.; et al. Are veterinary medicines causing environmental risks? Environmental Science and Technology, v. 37, n. 15, p. 286A294A, 2003.

BOXALL, A. B. A. The environmental side effects of medication. EMBO Reports, v. 5, n. 12, p. 1110-1116, 2004.

BOXALL, A.; LONG, C. Editorial - Veterinary medicines and the environment. Environmental Toxicology and Chemistry, v. 24, n. 4, p. 759-760, 2005.

BRACKETT, C. C.; SINGH, H.; BLOCK, J. H. Likelihood and mechanisms of cross-allergenicity between sulfonamide antibiotics and other drugs containing a sulfonamide functional group. Pharmacotherapy, v. 24, n. 7 I, p. 856-870, 2004.

BRASCHI, I.; BLASIOLI, S.; FELLET, C.; et al. Persistence and degradation of new $\beta$-lactam antibiotics in the soil and water environment. Chemosphere, v. 93, n. 1, p. 152-159, 2013.

CAPLETON, A. C.; COURAGE, C.; RUMSBY, P.; et al. Prioritising veterinary medicines according to their potential indirect human exposure and toxicity profile. Toxicology Letters, v. 163, n. 3, p. 213-223, 2006.

CARNEIRO, P.; KANEENE, J. B. Food inspection services: A comparison of programs in the US and Brazil. Food Control, v. 80, p. 314-318, 2017. 
CRIBB, A. E.; LEE, B. L.; TREPANIER, L. A.; et al. Adverse reactions to sulphonamide and sulphonamide-trimethoprim antimicrobials: Clinical syndromes and pathogenesis. Adverse Drug Reactions and Toxicological Reviews, v. 15, n. 1, p. 9-50, 1996.

DÍAZ-CRUZ, M. S.; GARCÍA-GALÁN, M. J.; BARCELÓ, D. Highly sensitive simultaneous determination of sulfonamide antibiotics and one metabolite in environmental waters by liquid chromatography-quadrupole linear ion trap-mass spectrometry. Journal of Chromatography A, v. 1193, n. 1-2, p. 50-59, 2008.

DONG, Z.; SENN, D. B.; MORAN, R. E.; et al. Prioritizing environmental risk of prescription pharmaceuticals. Regulatory Toxicology and Pharmacology, v. 65, n. 1, p. 60-67, 2013.

DREWS, J. Drug discovery: A historical perspective. Science, v. 287, n. 5460, p. 1960-1964, 2000.

FERRER, I.; ZWEIGENBAUM, J. A.; THURMAN, E. M. Analysis of 70 Environmental Protection Agency priority pharmaceuticals in water by EPA Method 1694. Journal of Chromatography A, v. 1217, n. 36, p. 5674-5686, 2010.

GARCÍA-GALÁN, M. J.; DÍAZ-CRUZ, M. S.; BARCELÓ, D. Combining chemical analysis and ecotoxicity to determine environmental exposure and to assess risk from sulfonamides. TrAC - Trends in Analytical Chemistry, v. 28, n. 6, p. 804-819, 2009.

GHIMIRE, S.; KYUNG, E.; LEE, J. H.; et al. An evidence-based approach for providing cautionary recommendations to sulfonamide-allergic patients and determining crossreactivity among sulfonamide-containing medications. Journal of Clinical Pharmacy and Therapeutics, v. 38, n. 3, p. 196-202, 2013.

GIBS, J.; HECKATHORN, H. A.; MEYER, M. T.; et al. Occurrence and partitioning of antibiotic compounds found in the water column and bottom sediments from a stream receiving two wastewater treatment plant effluents in Northern New Jersey, 2008. Science of the Total Environment, v. 458-460, p. 107-116, 2013.

HOFF, R. B.; BARRETO, F.; MELO, J.; et al. Characterization and estimation of sulfaquinoxaline metabolites in animal tissues using liquid chromatography coupled to tandem mass spectrometry. Analytical Methods, v. 4, n. 9, p. 2822-2830, 2012.

HOFF, R. B.; PIZZOLATO, T. M.; PERALBA, M.; et al. Determination of sulfonamide antibiotics and metabolites in liver, muscle and kidney samples by pressurized liquid extraction or ultrasound-assisted extraction followed by liquid chromatography-quadrupole linear ion trap-tandem mass spectrometry (HPLC-QqLIT-MS/MS). Talanta, v. 134, p. 768-778, 2015.

JI, K.; KIM, S.; HAN, S.; et al. Risk assessment of chlortetracycline, oxytetracycline, sulfamethazine, sulfathiazole, and erythromycin in aquatic environment: Are the current environmental concentrations safe? Ecotoxicology, v. 21, n. 7, p. 2031-2050, 2012. 
LEITE, M. P. M. B.; JOKL, L. Occurrence of sulfonamide residues in meat products from meat packing plants, inspected by the MAARA, during the period of 1990-1994. Boletim da Sociedade Brasileira de Ciencia e Tecnologia de Alimentos (Brazil), jun. 1996. Disponível em: <http://agris.fao.org/agris-search/search.do?recordID=BR9606840>. Acesso em: 18 dez. 2013.

LIN, T.; CHEN, Y.; CHEN, W. Impact of toxicological properties of sulfonamides on the growth of zebrafish embryos in the water. Environmental Toxicology and Pharmacology, v. 36, n. 3, p. 1068-1076, 2013.

MAURICIO, A. D. Q.; LINS, E. S.; ALVARENGA, M. B. A National Residue Control Plan from the analytical perspective-The Brazilian case. Analytica Chimica Acta, v. 637, n. 1-2, p. 333-336, 2009.

NEU, H. C. The crisis in antibiotic resistance. Science, v. 257, n. 5073, p. 1064-1073, 1992.

PARK, K.; KWAK, I. S. Gene expression of ribosomal protein mRNA in Chironomus riparius: Effects of endocrine disruptor chemicals and antibiotics. Comparative Biochemistry and Physiology - C Toxicology and Pharmacology, v. 156, n. 2, p. 113-120, 2012.

QUEIROZ MAURICIO, A.; LINS, E. S. The National Agricultural Laboratories of Brazil and the control of residues and contaminants in food. Food Additives \& Contaminants: Part A, v. 29, n. 4, p. 482-489, 2012.

SANTOS, L. H.; GROS, M.; RODRIGUEZ-MOZAZ, S.; et al. Contribution of hospital effluents to the load of pharmaceuticals in urban wastewaters: Identification of ecologically relevant pharmaceuticals. Science of the Total Environment, v. 461-462, p. 302-316, 2013.

SINDAN - SINDICATO NACIONAL DA INDÚSTRIA DE PRODUTOS PARA SAÚDE ANIMAL. 0 Setor - Mercado, 2016. Disponível em:

$<$ http://www.sindan.org.br/sd/base.aspx?controle=8>.

SINDAN - SINDICATO NACIONAL DA INDÚSTRIA DE PRODUTOS PARA SAÚDE ANIMAL. Compêndio de Produtos Veterinários, 2017. Disponível em:

<http://www.cpvs.com.br/cpvs/pesquisar.aspx>.

STRUCIŃSKI, P.; GÓRALCZYK, K.; CZAJA, K.; et al. Consumer risk assessment in case of maximum residue levels (MRLs) violations in food. Roczniki Państwowego Zakładu Higieny, v. 58, n. 2, p. 377-388, 2007.

VAN DER VEN, A. J. A. M.; VREE, T. B.; KOOPMANS, P. P.; et al. Metabolites and side effects of trimethoprim-sulfonamide combinations. Chemotherapie Journal, v. 6, n. 1, p. 28-30, 1997. 
VREE, T. B.; HEKSTER, Y. A.; LIPPENS, B. J. J. Clinical pharmacokinetics of sulfonamides in children: Relationship between maturing kidney function and renal clearance of sulfonamides. Therapeutic Drug Monitoring, v. 7, n. 2, p. 130-147, 1985.

VREE, T. B.; VAN DER VEN, A. J. A. M. Clinical pharmacokinetics of sulphonamides. Chemotherapie Journal, v. 6, n. 1, p. 21-27, 1997.

WALL, R.; STRONG, L. Environmental consequences of treating cattle with the antiparasitic drug ivermectin. Nature, v. 327, n. 6121, p. 418-421, 1987.

Autor para correspondência: Rodrigo Barcellos Hoff. Ministério da Agricultura, Pecuária e Abastecimento, LANAGRO-RS, Estrada da Ponta Grossa, no 3036, CEP.: 91780-580 - Porto Alegre-RS. rodrigo.hoff@agricultura.gov.br 